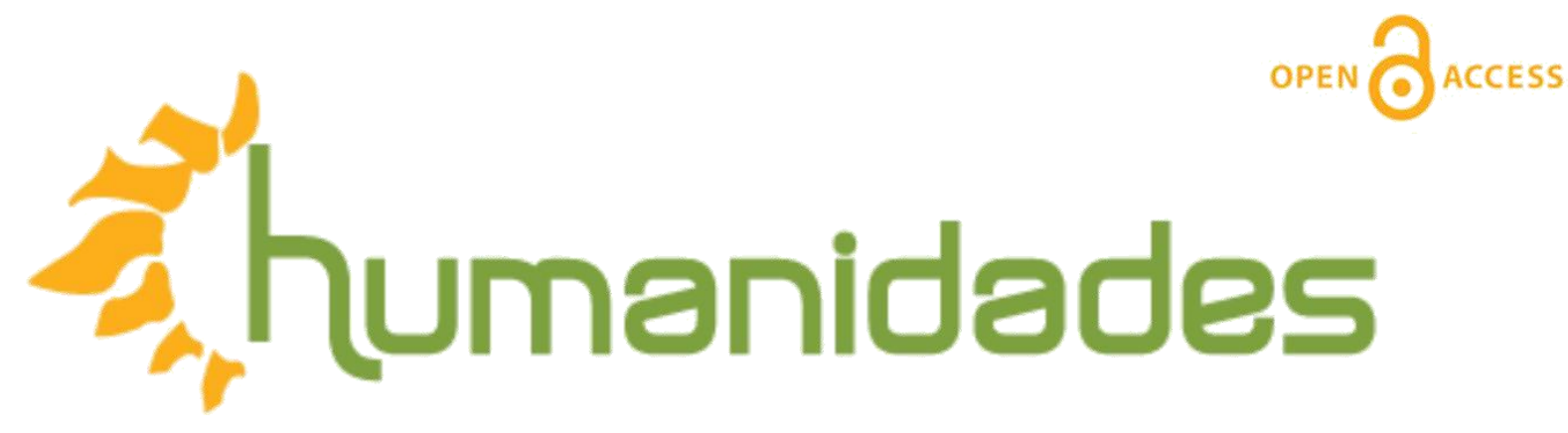

Revista de la Escuela de Estudios Generales, Universidad de Costa Rica

Julio - Diciembre, 2017 •Volumen 7, número 2 •EISSN 2215-3934•

Recibido: 13-Marzo-2017 Aceptado: 1-Junio-2017

\title{
El estudio de las presunciones en filosofía y lingüística: el testimonio de los diccionarios especializados
}

DOI: http://dx.doi.org/10.15517/h.v7i2.28191

\section{Javier Ramia}

Departamento de Filosofía. Universidad de Granada. ESPAÑA.

Correo electrónico: jramia@ugr.es

Todos los derechos reservados. Universidad de Costa Rica. Esta revista se encuentra licenciada con Creative Commons. Reconocimiento-NoComercial-SinObraDerivada 3.0 Costa Rica.

Correo electrónico: humanidades@ucr.ac.cr / Sitio web: http://revistas.ucr.ac.cr/index.php/humanidades 


\title{
El estudio de las presunciones en filosofía y lingüística: el testimonio de los diccionarios especializados ${ }^{1}$
}

\section{Resumen}

El objetivo de este artículo es analizar el tratamiento que se da a la voz presunción en los diccionarios de filosofía y lingüística, en la idea de que podrían ilustrar las vicisitudes del estudio de dicha noción en tales disciplinas. Se muestra que, aunque en las últimas décadas el estudio de las presunciones ha ocupado en buena medida a filósofos y teóricos de la argumentación, los diccionarios estudiados no les dedican apenas atención. Se analiza y comenta el contenido de cada entrada y se pone de manifiesto, de un lado, la evolución y las diferencias en los valores indicados y, de otro, la idoneidad de un tratamiento más extendido en (futuras ediciones de) dichas obras de referencia.

Palabras claves: presunción, filosofía, lingüística, diccionarios.

\section{The study on presumptions in philosophy and linguistics: the testimony of specialized dictionaries}

\begin{abstract}
The aim of this paper is to analyse the treatment of the term presumption in philosophical and linguistic dictionaries, on the idea that they may illustrate the vicissitudes of study of that notion in such disciplines. It is showed out that, even though in the last decades philosophers and argumentation theorists focused on the study on presumptions, the dictionaries studied do not pay much attention to that notion. The content of each entry is analysed and commented and it is indicated, firstly, the evolution and differences in the meanings and, secondly, the suitability of a more widespread treatment in (future editions of) such reference works.
\end{abstract}

Keywords: presumption, philosophy, linguistics, dictionaries.

${ }^{1}$ Este trabajo ha sido posible gracias a una Ayuda para Contratos Predoctorales para la Formación de Doctores concedida por el Ministerio de Economía y Competitividad y cofinanciada por el Fondo Social Europeo en el marco del proyecto de investigación de excelencia «El uso de las presunciones en el discurso argumentativo» (FFI201454681-P) 


\section{Introducción}

Aunque el origen de la teorización sobre las presunciones se encuentra en el campo del derecho, su estudio ha sobrepasado las fronteras de dicha disciplina. En efecto, ha habido acercamientos a la noción de presunción desde campos aparentemente tan dispares como la argumentación, la epistemología, la inteligencia artificial o la pragmática lingüística (Santibáñez 2010: 134; Gama: en prensa). Con todo, en casos como el que nos ocupa es preciso tener en mente un apunte expuesto por Allen (2014: 195) a propósito de la noción de carga de la prueba que, con alguna probabilidad, sería extrapolable al concepto de presunción: "as is often the case with disciplines reaching across boundaries, it is not at all clear that the term means the same thing".

Pues bien, aunque se trata de un concepto que se ha demostrado de interés en disciplinas distintas del derecho, no ha merecido examen de manera amplia hasta fechas recientes en dichos campos de estudio (Rescher 2003: 98). A este respecto, no es infrecuente encontrar, por ejemplo, comentarios de diversos autores que subrayan la poca atención que se ha prestado tradicionalmente a la noción de presunción en filosofía. En efecto, en un reconocido trabajo sobre las presunciones, Ullman-Margalit (1983: 143) señalaba que "the notion itself has not so far been the focus of proper philosophical attention”. Cummings (2010: 80), por su parte, sostenía que no hay razones para pensar que la situación haya cambiado desde el referido trabajo de Ullman-Margalit.

En esta senda, es doblemente interesante el apunte que hizo en su momento Walton (1993: 140), pues no sólo puso de manifiesto la poca atención que había prestado la filosofía a una noción de tanta relevancia como esta, sino que además introdujo otra variable, a saber, la comparación con la voz presuposición. En efecto, a diferencia de aquella, esta sí había despertado gran interés no sólo por parte de los filósofos, sino también de los lingüistas:

Presumption is a notion that is fundamental to philosophy as a subject, but has generally been ignored as a concept for serious investigation. In contrast, presupposition is a concept that has been studied in great intensity by both philosophers and linguists, resulting in a prolific variety of different theories, summarized by Levinson (1983).

Pues bien, a la luz de estas sugestivas referencias, en este trabajo nos proponemos analizar qué tratamiento se ha dado a las presunciones en filosofía y linguí́stica por medio del examen de diccionarios especializados de ambas disciplinas, en la idea de que, como detallamos a continuación, representarían una herramienta útil para analizar el estudio de dicha noción en tales campos de estudio durante un amplio espacio de tiempo.

\section{Los diccionarios como fuente de estudio}

En la medida en que nos proponemos estudiar el tratamiento que se ha dado al concepto de la presunción en dos disciplinas concretas, esto es, filosofía y lingüística, consideramos que los diccionarios especializados nos permitirán conocer, aun con las limitaciones inherentes al empleo únicamente de este tipo de fuentes, la situación y vicisitudes de su estudio en estos dos campos. En efecto, es de esperar que este tipo de trabajos nos permitan reconocer aspectos como la atención prestada a dicha noción, posibles variaciones o evoluciones de significado, así como sus distintos dominios de aplicación. 
¿Por qué filosofía y lingüística? Pues bien, se trata de dos disciplinas que, aunque desde perspectivas distintas, comparten intereses particulares. En efecto, si focalizamos en el caso de las presunciones, es preciso señalar que una disciplina eminentemente filosófica como la epistemología y, sobre todo, otra a medio camino entre la filosofía y la lingüística como la teoría de la argumentación ${ }^{2}$ se están ocupando de su estudio. De este modo, siguiendo el apunte de Walton, podemos esperar que la voz presunción sea, a la manera del término presuposición, analizada en diccionarios de ambos tipos, por más que el tratamiento pueda ser en diversos aspectos divergente.

Asimismo, la idea de que bajo las genéricas rúbricas de filosofía y lingüística podrían quedar reflejadas con menor o mayor precisión otras disciplinas - epistemología, argumentación, análisis del discurso, retórica, etc.- justificaría, al menos a nuestro juicio, todavía más su empleo. Finalmente -aunque pueda parecer un apunte trivial-existe una gran variedad de diccionarios de estas disciplinas que, con diferente grado de profundidad, presentan definiciones de términos pertenecientes a dichos campos de estudio. Así pues, esta pluralidad en número y especificidad nos habrá de permitir, en principio, disponer de un corpus en buena medida válido.

¿Por qué no empleamos diccionarios de derecho si la voz presunción tiene su origen en dicho campo? Pues bien, en la medida en que nos interesa el concepto de presunción más allá del derecho, no consideramos que sea primordial el repaso a dicha noción desde una perspectiva legal; asimismo, la cantidad de material de que dispondríamos convertiría nuestra labor en algo inabarcable en un artículo $^{3}$.

\subsection{Diccionarios de Filosofía}

Comenzamos nuestra labor con el examen del tratamiento que se ha dado al término presunción en los diccionarios de filosofía. Como hemos visto, son diversos los comentarios que pueden encontrarse en artículos y otros trabajos de investigación al respecto de la poca atención que se ha prestado desde la filosofía a esta noción. Pues bien, este hecho es fácilmente constatable por medio de un recorrido por los principales diccionarios generales de filosofía.

Con todo, queremos manifestar que no hemos analizado de forma exhaustiva la totalidad de diccionarios filosóficos $-\mathrm{y}$, más adelante, lingüísticos- existentes, sino que trabajamos sobre una selección de los mismos que aspira a ser considerada como suficientemente ilustrativa.

\footnotetext{
${ }^{2}$ A este respecto, van Eemeren et al. (2014: 29) apuntan que: "we therefore think that argumentation theory can best be viewed as an interdisciplinary study in its own right with logical, dialectical, and rhetorical dimensions that are to be nourished by the combined efforts of philosophers, logicians, linguists, discourse analysts, communication scientists, rhetoricians, psychologists, lawyers, and all others who have something to contribute that is theoretically pertinent".

${ }^{3}$ Para una visión general de la noción técnica en derecho remitimos a las entradas consagradas a la voz presunción en el recientemente publicado Diccionario del Español Jurídico (2016), en cuya elaboración han participado la Real Academia Española y el Consejo General del Poder Judicial.
} 


\subsubsection{Recorrido bibliográfico}

Pues bien, no aparece la entrada correspondiente al vocablo presunción en obras de reconocido prestigio y frecuentemente actualizadas cuales la Standford Encyclopedia of Philosophy o Routledge Encyclopedia of Philosophy. De igual manera, tampoco se encuentra en otros diccionarios como los de Audi (1999), Blackburn (2016), Borchert (2006), Brugger et al. (2014), Edwards (1967), Ferrater Mora (2010), Fieser y Dowden (2016), Jacob (1990), Mautner (2005), Mittelstraß (2008) o Ritter et al. (2010). Los diccionarios enciclopédicos en que sí se recoge la voz presunción representan, pues, una minoría. En efecto, solamente hemos encontrado una entrada dedicada a este término en los diccionarios generales de filosofía de Abbagnano (2004), Honderich (2005/2008), Lalande (1968) y Waibl y Herdina (1997).

Empezamos nuestra labor por el último de ellos: Waibl y Herdina (1997). Este caso tiene la particularidad de no tratarse de un diccionario al uso, sino de un glosario que ofrece las equivalencias de terminología filosófica en inglés y alemán. Así, encontramos la traducción o, si se prefiere, equivalencia entre Präsumption y presumption (1997: 304), así como su vinculación con términos cuales asunción, hipótesis, presuposición y suposición. Podría parecer que un diccionario de este cariz aportaría pocos datos significativos; sin embargo, pone de manifiesto una de las dificultades para la caracterización de un término de este tipo, a saber, la delimitación de las fronteras con otras nociones con las que en algunos casos parecería solaparse.

En efecto, la distinción entre esta suerte de nociones suele estar presente en gran parte de los estudios consagrados a las presunciones, ya sea en trabajos de perspectiva argumentativa como los de Walton (1992, 1993), Godden y Walton (2007) o Bermejo-Luque (2016), ya sea en aquellos fundamentados desde una visión eminentemente epistemológica como, por ejemplo, el de Rescher (2003). Asimismo, existen artículos como los de Llewelyn (1962) o Kauffeld (1995) dedicados desde diferentes perspectivas específicamente a esta cuestión. Con todo, trazar las fronteras de cada uno de estos conceptos no es una cuestión sencilla y, en este sentido, consideramos que todavía se debe avanzar en la investigación consagrada a este particular.

Así pues, la contribución del primer diccionario, además de su objetivo principal, a saber, la exposición de las equivalencias entre inglés y alemán, es reconocer el término presunción como una voz de interés filosófico y poner sobre la mesa la vinculación de esta noción con otros vocablos de sentido, al menos en parte, semejante.

A continuación nos detenemos a analizar la entrada que hallamos en el Vocabulaire technique et critique de la philosophie de Lalande ([1926] 1968: 821-822), que constituye una entrada al uso y reproducimos íntegramente:

PRÉSOMPTION, D. A. Vermuten; B. Präsumtion; C. Dünkel; E. Presumption; I. Presunzione.

Les présomptions sont définies par le Code civil, livre III, titre III, section III, art. 1349: «des conséquences que la loi ou le magistrat tire d'un fait connu à un fait inconnu»; définition visiblement trop large, puisqu'elle envelopperait la preuve rigoureuse d'un fait qui n'est pas immédiatement connu ou directement attesté. La présomption est proprement et d'une manière plus précise une anticipation sur ce qui n'est pas prouvé. D'où trois sens divergents, qui se rencontrent déjà dans le mot latin: 
A. Raisonnement par lequel on pose, en matière de fait, une conclusion probable, quoique incertaine. «Les présomptions qui ne sont point établies par la loi sont abandonnées aux lumières et à la prudence du magistrat, qui ne doit admettre que les présomptions graves, précises et concordantes.» Ibid. 1353., - Il n’y a là qu'une simple présomption.»

B. Action de tenir une affirmation pour vraie jusqu'a preuve du contraire, ou même dans certains cas, nonobstant la preuve du contraire. «La présomption légale est celle qui est attachée par une loi spéciale à certains actes ou à certains faits» (par ex. l'autorité de la chose jugée, les cas dans lesquels la loi déclare certains actes nuls, en tant que présumés faits en fraude, etc.). «La présomption légale dispense de toute preuve celui au profit duquel elle existe». Ibid., 1350, 1352. «La remise volontaire de la grosse du titre fait présumer la remise de la dette ou le payement, sans préjudice de la preuve contraire.» Ibid., 1283.

Dans ces deux premiers sens présomption se dit soi de l'acte de présumer, soit de la chose présumée. Cf. Leibniz, Discours de la conformité de la foi avec la raison, II 33.

C. Action de trop présumer de soi-même: confiance exagérée d'un individu en ses facultés ou ses forces.

Rad. int.; A. Konjekt, - aj.; B. Prezum.; C. Prezunt.

Tras aportar las traducciones del término presunción a otras lenguas, Lalande presenta la definición de presunción presente en el código civil francés. Afirma el autor que se trata de una definición demasiado amplia y sostiene que, de un modo más preciso, una presunción constituye una anticipación o previsión de algo todavía no demostrado y, a continuación, ofrece unas acepciones que estarían ya presentes en el valor del étimo latino praesumptio. En este punto, es preciso detenernos en dos aspectos de interés: de un lado, en el peso de la noción legal de presunción y, de otro, en la referencia a la etimología latina.

En cuanto al primero de los aspectos, esto es, el fundamento en la noción legal, debe tenerse presente que ese es su origen y se emplea aquí como garante de las dos primeras acepciones: A) "Razonamiento por el cual se da una conclusión probable si bien incierta"; B) "Acción de aceptar una afirmación por cierta hasta la prueba de su contrario o, incluso, en determinados casos, no obstante la prueba de su contrario". Por lo demás, se deja ver un aspecto clave también en la teorización sobre las presunciones: la recurribilidad; es decir, su mantenimiento hasta que nuevas evidencias muestren lo contrario. Como apunta Rescher (2003: 85), que una proposición cuente como una presunción es distinto a que cuente como una verdad aceptada, pues aquella representa únicamente una inclinación epistémica provisional y condicional hacia ella.

En lo referente a la mención de la voz latina esbozada por Lalande, es preciso distinguir entre dos posibilidades: que se refiera a la etimología -praesumptio, praesumere - "tomar de antemano" o que remita al valor técnico en retórica latina de la voz praesumptio, el cual puede definirse como "una figura de pensamiento [...] por la cual nos adelantamos a responder a cuestiones que pueden objetársenos o preguntársenos" (Luján Atienza 1999: 125). Parece ciertamente más plausible que se refiera específicamente a una cuestión de tipo etimológico, esto es, a la idea de aceptar una tesis o conclusión de antemano, pues esta concepción genérica daría cabida a los sentidos expuestos por el propio Lalande. 
Así pues, la presente entrada focaliza esencialmente en la noción de presunción en el marco legal. Con todo, se dejaría entrever la traslación a otros campos como la epistemología; en todo caso, debido a la naturaleza de la entrada, esta traslación quedaría en parte a la capacidad de analogía del lector.

Damos paso, a continuación, a la entrada que leemos en la versión española del diccionario de Abbagnano ([1963] 2004: 946), que cuenta -al igual que la obra anterior- hasta la fecha con un buen número de reediciones y reimpresiones:

Presunción (lat. praesumptio; ingl. presumption; fr. présomption; Alem. Präsumption; ital. presunzione). 1) Un juicio anticipado o provisional, que se considera como válido hasta prueba contraria. Por ejemplo, "P. de culpa" es un juicio de culpabilidad que se mantiene hasta en tanto no sea aducida una prueba contraria, y análoga significación tienen expresiones tales como "P. de verdad", "P. pro" o "P. contra" de una proposición cualquiera.

2) Confianza excesiva en las propias posibilidades y en este sentido se denomina presuntuoso al que nutre tal confianza.

Además de las equivalencias del término presunción en otras lenguas modernas, se ofrecen dos acepciones para la palabra que nos concierne. En la primera encontramos el apunte de que las presunciones constituyen un juicio provisional que se mantiene hasta que existe prueba que indique lo contrario; como hemos apuntado más arriba, la recurribilidad es uno de los elementos clave de las presunciones, aunque aquí no se indica la posibilidad de que una presunción se mantenga a pesar de la existencia de evidencias en su contra. Por lo demás, resulta cuando menos curioso que se hable de presunción de culpa pero no de inocencia. En este caso, aunque se observa la noción legal, esta ya no es fundamental y se dejaría acaso entrever el valor de las presunciones en epistemología.

El segundo de los valores -equiparable a la tercera de las acepciones en Lalande cuyo comentario hemos soslayado- parece alejarse de la noción que nos concierne. Sin embargo, diversos autores han reparado en la idea de presuntuosidad aunque de una forma distinta de la expresada en estos diccionarios. En efecto, Kauffeld (1995) la ha empleado para referirse a ciertos abusos en el empleo de las presunciones. De este modo, si alguien presume algo para lo que no está autorizado, se diría que tiene un comportamiento presuntuoso ${ }^{4}$.

Así pues, Abbagnano no se ocupa de ofrecer una suerte de introducción a la noción legal de presunción, sino de presentar de forma breve, según nuestro parecer, la idea subyacente aplicable más allá de los límites del derecho, esto es, la creación de juicios válidos recurribles. Por lo demás, aunque para la noción de presuntuoso, indicada también por Lalande, hemos señalado como paralelo lejano el apunte de Kauffeld, es preciso subrayar que tanto Lalande como el propio Abbagnano la emplean en su sentido no técnico.

El último de los diccionarios de filosofía en que nos detenemos es en el Oxford Companion to Philosophy de Honderich ([2005] 2008: 938-939). Pues bien, si comparamos esta obra con las demás, lo primero que debe subrayarse es que este diccionario constituye una excepción debido a que no

${ }^{4}$ Según Kauffeld (1995: 509-510), a diferencia de las asunciones, que representan "something one takes upon oneself", las presunciones suelen estar fundamentadas en aspectos sociales y, en ese sentido, una persona sólo puede presumir cuando se trata de "something to which he or she is entitled", en la idea de que "something is taken to be the case on the grounds that someone has or will have made that the case rather than risk resentment, criticism, reprobation, loss of esteem, or even punishment for failing to do so". 
recoge la voz presuposición y sí el término presunción. Sea como fuere, reproducimos a continuación la entrada correspondiente al término presunción en su versión española:

Presunción. Un concepto tomado del derecho por la epistemología que representa un modo de cubrir -al menos pro temp-ciertos huecos de nuestra información de otro modo asequible. La «presunción de inocencia» es aquí un caso paradigmático; la presunción es una cuestión no de hechos seguros, o de información dada, sino más bien de respuestas que damos por buenas en ausencia de indicaciones en contra. Las presunciones son según esto impugnables y vulnerables hasta poder ser derrocadas - pero sólo mediante ciertas consideraciones conflictivas debidamente establecidas-. Según esto, una presuposición posee por su parte una carga de prueba favorable: se necesita una sólida evidencia en contra para anular sus efectos.

La presuposición específica está siempre fundamentada en algún principio de presuposición. Tales principios operan sobre dominios diversos. Por ejemplo, una presuposición cognitiva opera a favor de los datos relevantes para la visión («Acepta lo que tú ves que es así»). En contextos de comunicación contamos con el presupuesto de que la gente es sincera («Acepta lo que tus interlocutores mantienen»); en nuestras indagaciones de la vida diaria nos movemos bajo la presuposición de que nuestras fuentes son fiables («Acepta lo que las enciclopedias y las autoridades dicen»); en ciencia nos apoyamos en el presupuesto de la suficiencia evidencial («Acepta lo que las teorías o explicaciones más rigurosamente fundadas establecer»). No obstante, el resultado de la aplicación de tales principios de presuposición es siempre tentativo y provisional: el aditamento «hasta el momento en que lo contrario se haga visible» es inseparable de todos los principios de presunción.

Las validaciones de estos principios pueden ser de dos tipos. Algunos de ellos están justificados a priori y proceden mostrando que son prerrequisitos sine qua non, de una determinada práctica cognitiva. Otros son a posteriori y de carácter experiencial, fundados en la consideración ex post facto de una grabación o registro que muestra que operar sobre esta base arroja mejores resultados para la empresa en cuestión que los que se conseguirían por otras vías. La eficacia práctica es lo esencial de la presuposición.

Las presuposiciones desempeñan un papel clave en las justificaciones cíclicas al uso en un coherentismo epistemológico que prescinde de las certezas auto-evidentes de un fundacionalismo epistémico.

N.R.

N. Rescher, Methodological Pragmatism, Oxford, 1977.

E. Ullman-Margalit, «On presumption», Journal of Philosophy, 1983.

Más allá de la comodidad inherente a la cita del texto en nuestra lengua, nos hemos decidido por tomar la definición de la versión española porque se produce un hecho ciertamente curioso: desde la parte final del primer párrafo se sustituye la voz presunción por presuposición. Este hecho no se da en la versión original inglesa y debe verse como una errata en la traducción; como reza el famoso adagio italiano, traduttore tradittore. Ya hemos señalado que se trata de conceptos con semejanzas y que pueden llevar a confusiones. ¿Habrá contribuido que no estuviese recogida la voz presuposición a que el traductor intercambiase de forma inconsciente un término por otro?

Dejando de lado este detalle, merece la pena señalar que en este caso estamos ante una entrada redactada por Rescher, uno de los autores cuyas contribuciones al estudio de las presunciones son valoradas y tomadas en consideración en nuevas propuestas y en los estados de la cuestión sobre esta 
noción. Con todo, este hecho podría llegar a verse en parte como un inconveniente, pues la disparidad de propuestas existentes sobre presunciones quedaría supeditada a sus propias propuestas.

En todo caso, en la senda de otros trabajos (Rescher 2003, por ejemplo) señala el autor que la presunción es un término tomado del campo del derecho por la epistemología y que tiene la función de "cubrir [...] ciertos huecos de nuestra información". Además de la mención explícita a la epistemología, este es el primer caso en que se señala para qué necesitamos esos juicios provisionales sujetos a recurribilidad, esto es, se indica cuál es la función -0 , más bien, una de ellas- de las presunciones. Asimismo, aunque Rescher toma la presunción de inocencia como caso paradigmático, vemos que, lejos de la entrada del diccionario de Lalande, la descripción de la noción legal no ocupa un papel primordial en la entrada.

Asimismo, se introduce por primera vez la noción de carga de la prueba, cuyo origen también se encuentra en el derecho y que el propio Rescher (2003: 83) considera en otro lugar un término "correlative with that of presumption". La idea general es que se necesitan razones sólidas para derribar una presunción; en otras palabras, la presunción tiene una carga de la prueba favorable. Tras ello, se señalan algunos principios de presunción y se exponen los dos tipos de validación de estos principios, a saber, a priori y a posteriori y se destaca el papel eminentemente práctico que, como recurso cognitivo, tienen las presunciones. Finalmente, se pone de manifiesto la función que ocupan en las justificaciones cíclicas, en la medida que permiten prescindir de certezas auto-evidentes.

La aportación principal de esta contribución es la de ir más allá, de dejar a un lado la noción estrictamente legal de presunción -aunque se use como paradigma la presunción de inocencia-para dar cuenta de dicho concepto desde una perspectiva epistemológica, explicando así, de un lado, qué papel juega en la creación de conocimiento y en los procesos de justificación y, de otro, su funcionamiento, naturaleza y fundamentos. Por lo demás, debe señalarse que es el primero de los trabajos mencionados en que se vincula explícitamente la presunción con la noción de carga de la prueba.

\subsubsection{Conclusiones parciales}

El tratamiento de las presunciones en los diccionarios de filosofía es en buena medida limitado. En efecto, solamente hemos podido hallar este término en cuatro de los diccionarios analizados y, además, hemos visto que en aquellos usualmente empleados como obras de referencia como, por ejemplo, la Standford Encyclopedia of Philosophy, no está consignado. Este hecho serviría, pues, para corroborar apuntes como los de Ullman-Margalit o Walton que hemos recogido al inicio del trabajo.

Asimismo, este examen nos ha permitido observar una sugestiva línea de evolución: desde la exposición de la noción estrictamente legal de presunción hasta la profundización en su papel más allá de del derecho, concretamente en el campo de la epistemología ${ }^{5}$. A este respecto, la entrada de Rescher representa un salto cualitativo en la atención prestada a este concepto.

${ }^{5}$ También es posible leer comentarios acerca de la poca atención a las presunciones concretamente en el campo de la epistemología: "presumption has been something of a poor cousin in epistemological discussion" (Cummings 2010: 57). 
Por lo demás, en las últimas fechas filósofos y teóricos de la argumentación han estudiado con profundo interés el fenómeno de las presunciones en el discurso argumentativo. Con todo, este valor no lo encontramos en diccionarios generales de filosofía. Dos razones podrían explicar este hecho: de un lado, que términos especializados de argumentación, considerada una disciplina independiente, no tengan cabida en diccionarios generales de filosofía; de otro, que los avances y generalización de su estudio se han producido en fechas recientes y, salvo los diccionarios que se reeditan con frecuencia, la mayoría de las obras mencionadas - aunque no todas- se publicaron con anterioridad a los referidos avances. Con todo, la primera posibilidad no sería del todo acertada pues, aunque vinculadas de modo general con cuestiones de lógica ${ }^{6}$, no es difícil hallar nociones que podríamos calificar de argumentativas en dichas obras de referencia.

\subsection{Diccionarios de Lingüística}

Si desplazamos nuestra labor a los diccionarios de linguiística, el resultado es, si cabe, todavía más negativo. Con todo, a diferencia de lo que ocurría en el caso de la filosofía, no hemos encontrado trabajos estrictamente de lingüística en que se ponga de manifiesto la poca atención prestada a esta noción. En todo caso, este hecho podría explicarse por una razón bien sencilla: para encontrar menciones de ese tipo sería necesario que hubiese trabajos de linguí́stica dedicados expresamente a esa noción.

\subsubsection{Recorrido bibliográfico}

Pues bien, mientras que el término presuposición está consignado en la mayoría de glosarios linguiísticos estudiados -excepto en Lázaro Carreter (1968) y Marouzeau (1951)-, no hay entrada para la voz presunción en los siguientes diccionarios: Abraham (1981), Alcaraz Varó y Martínez Linares (1997), Bussmann (1996), Cerdà Massó (1986), Crystal (1980, 1992), Dubois (1973), Frawley (2003), Lázaro Carreter (1968), Lewandoski (1982), Marouzeau (1951), Matthews (2014), Moreno Cabrera (1998), Pikabea Torrano (2008), Richards y Schmidt (2013), Trask (1997) y Welte (1985).

De hecho, solamente hemos encontrado este concepto en dos diccionarios lingüísticos. Por su naturaleza, la información que ofrecen es muy limitada y, además, los sentidos expresados son sensiblemente distintos no sólo a los ofrecidos por los diccionarios de filosofía sino también entre ellos mismos. Los trabajos en cuestión son los de Cardona (1991) y Sánchez Puig (1994).

En el primero de estos trabajos no hallamos sensu stricto el término que nos ocupa, sino un adjetivo derivado del mismo que su autor considera equivalente a inferencial:

Presuntivo. Aspecto verbal, del tipo Se habrá convertido ya en un gigante $\rightarrow$ inferencial. Inferencial. Aspecto verbal: Ya tendría que estar cocido $\rightarrow$ presuntivo.

Nos encontramos ante una utilización distinta de nuestro vocablo, ya que aquí hace referencia específica al aspecto verbal. Lázaro Carreter (1984: 63-64) definía el aspecto como el conjunto de

\footnotetext{
${ }^{6}$ La lógica fue el campo con el que hasta los años 50 del siglo pasado se asimilaba el estudio de la argumentación. Con todo, ya desde autores como Toulmin o Perelman y Olbrechts-Tyteca se la consideró insuficiente para tratar con la argumentación tal como acontece en la práctica real (van Eemeren et al. 2014: 141).
} 
"matices no temporales del desarrollo de la acción verbal que evocan las diversas formas verbales" y sostenía que se trata de "una de las nociones más difíciles y debatidas de la lingüística actual". De este modo, los dos ejemplos expuestos representarían conclusiones de inferencias basadas en eventuales indicios, cuya certeza es tentativa y estarían sujetos a recurribilidad. Así pues, aunque las ideas generales que subyacen al concepto parecen ser a grandes rasgos análogas a las presentes en el campo de la filosofía, su uso, como vemos, es muy específico, pues se situaría a un nivel equivalente a otros aspectos verbales como el perfectivo, imperfectivo o durativo.

Concluimos nuestro repaso bibliográfico de la misma manera que lo empezamos: con un glosario bilingüe. Ahora bien, en este caso no se trata de un trabajo en que se dan las equivalencias terminológicas entre inglés y alemán como en el de Waibl y Herdina (1997), sino que estamos ante un glosario español-ruso y ruso-español. Nos referimos, en efecto, al Diccionario de términos lingüísticos de Sánchez Puig (1994: 512), del que reproducimos la entrada en cuestión omitiendo las traducciones al ruso, pues lo que nos interesa son los términos vinculados:

Presunción (anticipación, catáfora, ocupación, prolepsis)

Pues bien, recordemos que en el diccionario de Waibl y Herdina (1997) se consignaban, junto a la voz presunción, conceptos como los de asunción, hipótesis, presuposición y suposición. Tratamos de explicar el porqué de ese hecho por medio de las dificultades inherentes a la distinción de términos que, en algunos casos, podrían llegar a verse como equivalentes ${ }^{7}$. Sin embargo, ahora nos encontramos con vocablos en buena medida distintos como anticipación, catáfora, ocupación y prolepsis. ¿A qué obedece este cambio de términos vinculados?

La idea que parece subyacer a estas nociones remitiría no sólo al origen etimológico del término presunción -praesumptio, praesumere- cuyo significado, en palabras de Gama (2013: 67), era "tomar, asumir o suponer anticipadamente", sino también -y más específicamente- al valor técnico de praesumptio en retórica clásica: la anticipación a posibles contraargumentos. Ello explica, pues, la presencia de términos relacionados como anticipación en vez de voces como asunción o hipótesis. En la misma senda se inscribe el resto de términos relacionados: catáfora se entiende como la relación de identidad entre un elemento y una palabra o grupo de palabras que se nombran más adelante en el discurso y, finalmente, tanto ocupación como prolepsis ${ }^{8}$ son términos técnicos en el campo de la retórica clásica y se refieren, con sus respectivos matices, a la anticipación por parte del autor a objeciones o argumentos que otro pudiera producir en su contra.

\subsubsection{Conclusiones parciales}

La voz presunción no recibe apenas tratamiento en los diccionarios de lingüística, hasta el punto de que su presencia en estas obras de referencia puede verse como un hecho prácticamente anecdótico. Este hecho, en suma, viene a corroborar que las presunciones no han sido, al menos de modo general, objeto de estudio de la linguiística. Por lo demás, la información contenida por estas obras diverge tanto entre ellas como en comparación con los diccionarios de filosofía, ya sea por su valor específico,

\footnotetext{
${ }^{7}$ Walton (1993: 140) a propósito de las presunciones y presuposiciones afirma que "both concepts can be put to use for varying purposes and there is some flexibility in how they can be interpreted. Consequently, it is not easy to tell the two apart, or to firmly fix their key differences. It seems that, in many cases, they refer to roughly the same thing".

${ }^{8}$ De hecho, según noticia de Quintiliano (Inst. 9.2.16.4: quae prolepsis dicitur), los griegos denominaban prolepsis al artificio retórico llamado en latín praesumptio.
} 
ya sea por su significado distinto. Con todo, los datos obtenidos deben ser tomados con mucha cautela, ya que los ejemplos con que contamos son mínimos.

En todo caso, no debería sorprender que una noción de este tipo que, como apuntamos, está siendo tratada en las últimas fechas con mucha atención por la filosofía y la teoría de la argumentación, se instale primero en estas disciplinas y, una vez asentada, se traslade al campo de la lingüística ${ }^{9}$. Es posible ilustrar esta conjetura con algunos paralelos: por ejemplo, la voz presuposición. En efecto, aunque se trata de una noción que, como comentaba el propio Walton, ha sido tratada tanto por filósofos como por lingüistas, su origen es eminentemente filosófico. Del mismo modo, otras nociones que se han considerado claves en lingüística como, por ejemplo, las de acto de habla o implicatura surgieron en el campo de la filosofía y, más tarde, fueron adoptadas por los estudios lingüísticos ${ }^{10}$. De hecho, incluso el campo de la pragmática, de gran interés para la lingüística, hunde sus raíces en la filosofía y sólo más tarde los lingüistas la tomaron no como algo ajeno, sino como una disciplina eminentemente propia.

\section{Conclusiones}

A lo largo de este trabajo hemos estudiado qué tratamiento se da a la voz presunción en los diccionarios de filosofía y lingüística mediante la compilación y comentario de las entradas correspondientes. Con todo, no está de más recordar, de un lado, que, aunque hemos tratado de atender a un número suficientemente ilustrativo de diccionarios, nuestro repaso no ha sido exhaustivo $\mathrm{y}$, de otro, que las consideraciones que hemos añadido se han limitado esencialmente a aquellos aspectos atendidos ya directa, ya indirectamente en dichos trabajos.

Este recorrido por diccionarios de filosofía y lingüística nos ha permitido corroborar apuntes como los de Ullman-Margalit (1983: 143), Walton (1993: 140) y Cummings (2010: 57, 80), a saber, que tradicionalmente las presunciones no han recibido apenas atención en el campo de la filosofía y, añadimos nosotros, en el de la lingüística. Un caso distinto, como leíamos en el mismo apunte de Walton y como hemos podido comprobar, lo constituyen las presuposiciones, tratadas, debido a la relevancia de su estudio desde tiempo atrás, por prácticamente la totalidad de diccionarios de ambas disciplinas.

Asimismo, hemos señalado las diferentes acepciones que se da a la voz presunción en estas obras de referencia, las cuales ponen de manifiesto los diferentes sentidos con que se ha usado este término. En efecto, hemos observado que los valores eran diferentes en los diccionarios de filosofía y lingüística y que en los primeros se dejaba ver el paso de la noción legal a la epistemológica. En este sentido, destacaba cualitativamente la entrada redactada por Rescher, en la medida en que era la única que proporcionaba un examen detallado más allá de la exposición de la noción eminentemente legal. Asimismo, es también digno de mención cómo la noción de presunción que hallamos en los glosarios filosóficos se aplicaba a un elemento muy concreto en un diccionario lingüístico: el aspecto verbal presuntivo. Del mismo modo, resultaba llamativo también el valor señalado por un glosario bilingüe

\footnotetext{
${ }^{9}$ Sobre todo, en la última de ellas, pues, aunque la noción estrictamente epistemológica tal vez no sería de su interés, la argumentativa - con muchos puntos en común con aquella - sí ocuparía, a la manera de otras nociones, a la lingüística.

${ }^{10}$ En Álvarez y Ginoria (1996: 29-32) puede verse un breve repaso histórico de la adopción de estas nociones originalmente filosóficas por parte de la lingüística.
} 
de términos lingüísticos, ya que nos retrotraía siglos atrás al indicar un significado presente en la retórica clásica.

Por lo demás, no hemos encontrado el desarrollo del valor argumentativo de la voz presunción en las obras estudiadas. Este hecho, como hemos apuntado, podía deberse a diversos factores: de un lado, la hipótesis poco precisa de que los diccionarios generales de filosofía o linguística no toman en consideración cuestiones de tipo argumentativo y, de otro, la más verosímil posibilidad de que el auge en los estudios sobre presunciones por parte de filósofos y teóricos de la argumentación se ha producido en fechas recientes, anteriores a la edición de gran parte de dichas obras de referencia.

Concluimos nuestro trabajo, en suma, manifestando la idoneidad de que en las próximas ediciones de los diccionarios de filosofía y, argumentación mediante, lingüística, así como en nuevos trabajos de referencia de estas disciplinas se dedique una entrada a la voz presunción en que se puedan observar los diversos aspectos que la caracterizan, unos aspectos que hacen de este concepto una noción de relevancia tanto para filósofos como para lingüistas ${ }^{11}$.

${ }^{11}$ Sirva, como fundamento de esta consideración, el apunte preliminar de Bermejo-Luque (2016: 1-2): "Philosophers have also paid attention to presumptions as devices for reaching conclusions under uncertainty. In this nontechnical sense, presumptions exceed the realm of law, playing a widespread cognitive role in both everyday and scientific reasoning. Contemporary philosophers and argumentation theorists working on presumptions like Nicholas Rescher $(1977 ; 2006)$, Douglas Walton $(1992 ; 1993 ; 1996 ; 2008)$ and James Freeman (2005) even contend that presumptions are unavoidable points of departure for any inquiry, and thus, conditions of possibility for achieving justification for our claims and beliefs. For, on the one hand, presumptions would articulate the exemption of providing further reasons for our reasons, which is something necessary if chains of reasoning are to stop at some point. And regarding argumentative exchanges, presumptions would serve to allocate the burden of proof among discussants, determining the path for a correct argumentative discussion to take place". 
El estudio de las presunciones...

\section{Referencias}

Abbagnano, N. (2004). Diccionario de Filosofía. actualizado y aumentado por G. Fornero (4 ${ }^{\mathrm{a}}$. ed.) (trad. J.E. Calderon et al.). México: Fondo de Cultura Económica.

Abraham, W. (1981). Diccionario de terminología lingüística actual. (trad. F. Meno Blanco). Madrid: Gredos.

Alcaraz Varó, E. y Martínez Linares, M.A. (1997). Diccionario de lingüística moderna. Barcelona: Ariel.

Allen, R.J. (2014). Burdens of proof. Law, Probability and Risk, 3(3-4), 195-219.

Álvarez, M. y Ginoria, M. (1996). Aproximación al concepto de «presuposición». Anuario de Estudios Filológicos, 19, 27-37.

Audi, R. (1999). The Cambridge dictionary of philosophy (2 $2^{\mathrm{a}}$. ed.). Cambridge: Cambridge University Press.

Bermejo-Luque, L. (2016). Being a Correct Presumption vs. Being Presumably the Case. Informal Logic, 36(1), 1-25.

Blackburn, S. (2016). The Oxford Dictionary of Philosophy. Oxford: Oxford University Press.

Borchert, D.M. (ed.) (2006). The Encyclopedia of Philosophy [2 $2^{\mathrm{a}}$. ed.]. Farmington Hills: Thomson Gale.

Brugger, W. et al. (2014). Diccionario de filosofía. [trad. R. Gabás]. [2ª . ed.]. Barcelona: Herder.

Bussmann, H. (1996). Routledge Dictionary of Language and Lingüistics [trad. y ed. G. Trauth y K. Kazzazi]. London and New York: Routledge.

Cardona, G.R. (1991). Diccionario de lingüística. [trad. Ma T. Cabello]. Barcelona: Ariel.

Cerdà Massó, R. et al. (coord.) (1986). Diccionario de Lingüística. Madrid: Anaya.

Craig, E., (ed.) (2016). Routledge Encyclopedia of Philosophy. Version online: https://www.rep.routledge.com.

Crystal, D. (1980). A Dictionary of Linguistics and Phonetics. Cambridge / Massachusetts: Blackwell.

Crystal, D. (1992). An Encyclopedic Dictionary of Language and Languages. Cambridge / Massachusetts: Blackwell.

Dubois, J. et al. (1973). Dictionnaire linguistique. París: Larousse.

Edwards, P. (ed.) (1967). The Encyclopedia of Philosophy. Nueva York: Macmillan. 
Eemeren, van F.H et al. (2014). Handbook of Argumentation Theory. Dordrecht: Springer.

Ferrater Mora, J. (2010). Diccionario de Filosofía. [6 ${ }^{\mathrm{a}}$. ed.] Madrid: Alianza.

Frawley, W.J. (2004). International Encyclopedia of Linguistics [2 ${ }^{\mathrm{a}}$. ed.]. Oxford: Oxford University Press.

Freeman, J. (2005). Acceptable Premises. Cambridge: Cambridge University Press.

Gama, R. (2013). Concepciones y tipología de las presunciones en el derecho continental. REJ, 19, 65-89.

Gama, R. (en prensa). The nature and place of presumptions in law and legal argumentation. Argumentation.

Honderich, N.R. (2005). Oxford Companion to Philosophy [2a . ed.]. Oxford: Oxford University Press [trad. española = (2008). Enciclopedia Oxford de Filosofía. Madrid: Tecnos].

Kauffeld, A. (1995). On the Difference Between Assumptions and Presumptions. En S. Jackson (ed), Argumentation and Values, Annandale, VA: Speech Communication Association, 509-514.

Jacob, A. (dir) (1990). Encyclopédie Philosophique Universelle, vol. II: Les notions philosophiques, Tome II, Philosophie Occidentale (M-Z). París: Presses Universitaires de France.

Lalande, A. (1968). Vocabulaire technique et critique de la philosophie. [10 ed.]. París: Presses Universitaires de France.

Lázaro Carreter, F. (1968). Diccionario de términos filológicos. Madrid: Gredos.

Lewandoski, T. (1982). Diccionario de lingüística [trad. Ma . L. García-Denche y E Bernárdez]. Madrid: Cátedra.

Llewelyn, J.E. (1962). Presuppositions, assumptions and presumptions. Theoria, 28, 157-172.

Luján Atienza, A.L. (1999). Retóricas españolas del siglo XVI. El foco de Valencia. Madrid: Consejo Superior de Investigaciones Científicas.

Marouzeau, J. (1951). Lexique de la terminologie linguistique français, allemand, anglais, italien $\left[3^{\mathrm{a}}\right.$. ed.]. París: Librairie Orientaliste Paul Geuthner.

Matthews, P. (2014). The Concise Oxford Dictionary of Linguistics. [3 ${ }^{\mathrm{a}}$. ed.]. Oxford: Oxford University Press.

Mautner, T. (2005). The Penguin dictionary of philosophy [2a . ed.]. Londres: Penguin books.

Mittelstraß, J. (2008). Enzyklopädie Philosophie und Wissenschafttheorie. Stuttgart/Weimar: Metzler. 
El estudio de las presunciones...

Moreno Cabrera, J.C. (1998). Diccionario de lingüística neológico y multilingüe. Madrid: Síntesis.

Muñoz Machado, S. [Real Academia Española; Consejo General del Poder Judicial] (dir.) (2016). Diccionario del Español Jurídico. Madrid: Espasa Libros.

Pikabea Torrano, I. (2008). Glosario del lenguaje. La Coruña: Netbiblo.

Rescher, N. (1977). Dialectics: A Controversy-Oriented Approach to the Theory of Knowledge. Albany, NY: State University of New York Press.

Rescher, N. (2003). Epistemology: An Introduction to the Theory of Knowledge. Albany: State University of New York.

Rescher, N. (2006). Presumption and the Practices of Tentative Cognition. Cambridge: Cambridge University Press.

Richards, J.C. y R.W. Schmidt (2013). Longman Dictionary of Language Teaching and Applied Linguistics ( $4^{\mathrm{a}}$. ed.). Routledge: New York.

Ritter, J., et al. (2010). Historisches wörterbuch der philosophie. CD-Rom.

Sánchez Puig, M. (1994). Diccionario de términos lingüísticos: ruso-español y español-ruso. Madrid: Universidad Complutense de Madrid.

Trask, L., (1997). A Student's Dictionary of Language and Linguistics. London and New York: Routledge.

Ullmann-Margalit, E., (1983). On Presumption. The Journal of Philosophy, 80(3), 143-163.

Waibl, E.; Herdina, P. (1997). Dictionary of philosophical terms = Wörterbuch philosophischer Fachegriffe. Múnich: K.G. Saur.

Walton, D. (1992). Plausible Argument in Everyday Conversation. Albany: State University of New York Press.

Walton, D. (1993). The Speech Act of Presumption. Pragmatics \& Cognition, 1, 125-148.

Welte, W. (1985). Lingüística moderna. Terminología y bibliografía [trad. F. Meno Blanco]. Madrid: Gredos.

Whately, R. [1846] (1963). Elements of Rhetoric (7th ed. D. Ehninger). Carbondale: Southern Illinois University Press.

Zalta, E.N. (ed.). Standford Encyclopedia of Philosophy. Recuperado de: https://plato.stanford.edu. 


\section{¿Cómo citar este artículo?}

Ramia, J. (2017). El estudio de las presunciones en filosofía y lingüística: el testimonio de los diccionarios especializados. Revista humanidades, 7(2). doi:http://dx.doi.org/10.15517/h.v7i2.28191 